\title{
Subcutaneous Sparganosis: A Rare Case Report
}

\author{
Dr. Anusha A.M., M.D1 \\ ${ }^{I}$ Department of Pathology, Dr. Pinnamaneni Siddhartha Institute of Medical Sciences \& Research Foundation, \\ Chinoutapalli,Andhra Pradesh, India
}

\begin{abstract}
Human sparganosis is a rare parasitic disease as a result of infection by plerocercoid larva (sparganum) of Spirometra species. In this case report, a 31-year-old man presented as a subcutaneous nodule in the axilla for 5 months, which was excised. Characteristic plerocercoid larvae accompanied by sinus tract and abscess in the subcutaneous tissue were discovered under microscopic examination of the excised tissue. This report aims to highlight the existence and presentation of sparganum infection which is considered to be rare in Indian subcontinent. Also this case report reveals an atypical picture where the larval forms was not identified on gross examination of the excised tissue and the diagnosis was confirmed on the basis of microscopy.
\end{abstract}

Keywords: Sparganosis, sparganum, spirometra, rare parasitic disease, subcutaneous nodule

\section{Introduction}

The Cestode Spirometra is usually of no direct harm to human beings. It's the plerocercoid larva (sparganum), which is of significance from the pathological perspective. Sparganosisis transmitted by the ingestion or contact of humans with the intermediate hosts which includes copepods (crustaceans of the genus Cyclops) and the amphibians, reptiles that consume the copepods which harbor the larva (sparganum)of spirometra. Human beings are accidental dead-end host [1] [2]

Sparganosis is extremely rare in its prevalence across the globe while in having endemic presence in some geographical regions like south east Asia and countries like Japan, Korea and China [3], since only 450 cases have been reported worldwide and probably only 7 cases from India the need for its awareness is essentialfor timely diagnosis and intervention [4] [5]

The knowledge about the prevalence of sparganosis beyond the borders of its endemic area adds to the armamentarium of differential diagnosis and timely therapeutic interventions.Literature review signifies surgical access and gross examination of the affected area and visualization of the larval forms as a routine diagnostic practice while other methods like ELISA,MRI, CT put on surrogate status.This study has highlighted the significance of microscopic examination in diagnosis of sparganosis.

\section{Case Report}

A 31-year-old male patient, resident of Andhra Pradesh, presented with axillary swelling of 5 months duration. Family history was unremarkable. He had a pet dog. On physical examination the patient was conscious and well oriented with vital parameters in the normal limits.There was a raise in the erythrocyte sedimentation rate to $80 \mathrm{~mm}$ in the $1^{\text {st }}$ hour. Other biochemical and hematological parameters were within normal limits. The patient was advised surgical excision of the mass.

The axillary mass was excised and sent to the Department of Pathology of Dr. Pinnamaneni Siddhartha Institute of Medical Sciences \& Research Foundation, Chinoutapalli, Andhra Pradesh. The specimen was already cut open, measuring $3 \times 2 \times 1.5 \mathrm{~cm}$. External surface was gray white. Inner surface showed gray white and necrotic areas.

Microscopy of transverse sections revealeda segment of broad solid non cavitatory body of a plerocercoid larva (figure 1), while longitudinal section showedcharacteristic longitudinal muscles in the wall (figure 2). Other areas showed sinus tracts and abscess formation (figure 3).No protoscolex or scolex was seen. 




\section{Discussion}

On gross examination of the resected tissue mass, necrotic areas was noted, but no larval forms could be identified. It was only in the microscopic examination that the larval form was seen and the diagnosis of sparganosis concluded.

The definitive hosts of the life cycle are cats and dogs while Humans are infected accidently,as the patient had a pet dog, infection from the dog could be the possibility. The differential diagnosis of taenia solium could be ruled out by considering a key difference which is the absence of a protoscolex or scolex in Spirometra .Extreme rarity of sparganosis and lack of knowledge about its existence can result in delayed diagnosis and later therapeutic interventions which will add on to the sufferings of the patient.[7] Sparganosis as a usual practice is diagnosed by the surgical removal of the worm from the site of inflammation but in cases where there is a limited feasibilityof surgery, surrogate diagnostic means like antisparganum ELISA tests in tune with a relevant history of exposure can be used [2] but this case report where gross examination of the excised tissue did not reveal any larval forms, it was the microscopic study which helped in the diagnosis of sparganosis.

\section{References}

[1]. Garcia, L., and Bruckner, D.A. Diagnostic Medical Parasitology. Herndon, VA: ASM Press, 2007

[2]. Walker, M.D., and Zunt, Z.R.."Neuroparasitic Infections: Cestodes, Trematodes, and Protozoans.” Seminars in Neurology 25.3 (2005): 262-277.

[3]. Apatow SM. Humanitarian Resource Institute Biodefense Reference Library Foreign Animal and Zoonotic Disease Center One Medicine: One Health (Zoonotic Disease) Online Course. University of California, Santa Barbara. http://www.humanitarian.net/biodefense/fazdc/zdc1/zoores_cestode.html.

[4]. Song, T., Wang, W.S., and W.W. Mai. "CT and MR Characteristics of Cerebral Sparganosis.” AJNR. 28 (2007): 1700-1705.

[5]. Rengarajan S, Nanjegowda N, Bhat D, Mahadevan A, Sampath S, and Krishna S: Cerebral sparganosis: a diagnostic challenge. Br J Neurosurg; (2008) Dec;22(6):784-6

[6]. Mukherjee B, Biswas J, Raman M. Subconjunctival larva migrans caused by sparganum. Indian J Ophthalmol(2007) ;55:242-3

[7]. Duggal S, Mahajan RK, Duggal N, Hans C. Case of sparganosis: A diagnostic dilemma. Indian J Med Microbiol(2011) ;29:183-6

[8]. Iwatani, K., Kubota, I., Hirotsu, Y., et al. "Sparganum mansoni parasitic infection in the lung showing a nodule." Pathology International. 56(11): 674-7. 